\title{
Mapeando os estudos sobre educação médica no Brasil: Tendências e perspectivas
}

\author{
Eveline Tonelotto Barbosa Pott \\ Psicóloga, Mestre e Doutora em Psicologia. \\ Centro Universitário de Jaguariuna (UniFAJ) \\ 凶 evelinebarbosaa@gmail.com \\ Henrique Pott Junior \\ Médico e Doutor em Infectologia. Departmento de Medicina. \\ Universidade Federal de São Carlos (UFSCar) \\ 凶henriquepott@ufscar.br
}

Aceito em 15 de maio de 2019

\begin{abstract}
Resumo:
Este estudo tem como objetivo mapear os principais temas estudados na área da Educação Médica ao nivel da graduação, possibilitando traçar as principais tendências e propostas pedagógicas para a formação do profissional médico no Brasil. Para tanto, foi realizado uma revisão dos estudos publicados de 2013 à 2018, utilizando-se como descritores "educação médica" e "educação de graduação em medicina". Um total de 93 estudos foram analisados e organizados nas seguintes categorias de anáise: a arte mobilizando o ensino; o processo de avaliação; a formação e as percepções de docentes; o uso de tecnologias digitais no ensino médico; as percepções de alunos sobre o ensino médico; metodologias ativas de ensinoaprendizagem; relatos de experiência de instituições de ensino médico sobre a reformulação curricular; relatos de experiência sobre a reformulação de disciplinas; e o repensar sobre a educação médica. Como resultados do estudo, constatou-se que os principais temas de investigação na área de Educação Médica referem-se ao uso de tecnologias digitais mobilizando o ensino e as metodologias ativas de ensino e aprendizagem. Por outro lado, os temas que menos apresentaram estudos foram aqueles referentes ao processo de avaliação e a percepção de alunos e professores acerca nas inovações curriculares. Evidencia-se, assim, que há uma tendência em criar novas estratégias de ensino, porém faltam estudos que avaliem os impactos da implementação destas estratégias sob a perspectiva de docentes e discentes. Com este estudo, foi possível caracterizar os principais temas, mapeando as tendências e projetando perspectivas de futuras investigações na área de Educação Médica.

Palavras-chave: Educação médica, Educação de graduação em medicina, Revisão sistemática.
\end{abstract}

\section{Mapping medical education research in Brazil: trends and perspectives}

\begin{abstract}
:
This review aimed to synthesize the literature describing the main subjects studied in the area of undergraduate medical education, making it possible to trace the main tendencies and pedagogical proposals for the training of medical professionals in Brazil. A systematic review was performed of articles published between 2013 and 2018, using as descriptors "medical education" and "undergraduate medical education". A total of 93 studies were analyzed and organized in the
\end{abstract}


following categories: art mobilizing teaching; the evaluation process; teacher training and perceptions; the use of digital technologies in medical education; student perceptions of medical education; active teaching-learning methodologies; experience reports of medical teaching institutions on curriculum reformulation; experience reports on discipline's reformulation; and rethinking about medical education. As a result of the study, it was found that the main research topics in the area of Medical Education refer to the use of digital technologies mobilizing teaching and active teaching-learning methodologies. On the other hand, the subjects that least presented studies were those referring to the evaluation process and the perception of students and teachers about curricular innovations. Thus, there is a tendency to create new teaching strategies, but there is a lack of studies that evaluate the impacts of the implementation of these strategies from the perspective of teachers and students. With this study, it was possible to characterize the main subjects, mapping the trends and projecting perspectives of future investigations in the area of Medical Education.

Keywords: Medical education, Undergraduate medical education, Systematic review.

\section{Mapeo de la investigación en educación médica en Brasil: tendencias y perspectivas}

\section{Resumen:}

Esta revisión tuvo como objetivo sintetizar la literatura que describe los principales temas estudiados en el área de la educación médica de pregrado, lo que permite rastrear las principales tendencias y propuestas pedagógicas para la formación de profesionales médicos en Brasil. Se realizó una revisión sistemática de los artículos publicados entre 2013 y 2018, utilizando como descriptores "educación médica" y "educación médica de pregrado". Un total de 93 estudios fueron analizados y organizados en las siguientes categorías: arte movilizando la enseñanza; el proceso de evaluación; formación docente y percepciones; el uso de las tecnologías digitales en la educación médica; percepciones de los estudiantes de la educación médica; metodologías activas de enseñanza-aprendizaje; informes de experiencia de instituciones de enseñanza médica sobre reformulación curricular; informes de experiencias sobre la reformulación de la disciplina; y repensar sobre la educación médica. Como resultado del estudio, se encontró que los principales temas de investigación en el área de Educación Médica se refieren al uso de tecnologías digitales que movilizan la enseñanza y las metodologías activas de enseñanza-aprendizaje. Por otro lado, los sujetos que menos presentaron estudios fueron los que se refieren al proceso de evaluación y la percepción de los estudiantes y profesores sobre las innovaciones curriculares. Por lo tanto, hay una tendencia a crear nuevas estrategias de enseñanza, pero hay una falta de estudios que evalúen los impactos de la implementación de estas estrategias desde la perspectiva de docentes y estudiantes. Con este estudio, fue posible caracterizar los temas principales, mapear las tendencias y proyectar perspectivas de investigaciones futuras en el área de Educación Médica.

Palabras clave: Educación médica, Educación médica de pregrado, Revisión sistemática.

\section{INTRODUÇÃO INTRODUÇÃO}

Devido às transformações econômicas e sociais em nossa sociedade, cada vez mais tem se exigido da educação superior a formação de um novo perfil profissional, fundamentado em conceitos científicos, técnicos e também em um conjunto de competências importantes para a atuação profissional, como por exemplo: o trabalho em equipe, o cuidado ético na prática profissional, a responsabilidade social, e o 
comprometimento com o estudo contínuo, entre outras que transcendem a dimensão unicamente técnica (DIAS et al., 2018).

Em especial no que concerne à educação médica, inúmeros são os desafios que permeiam o processo de formação do médico no Brasil e no mundo. á décadas o ensino médico vem sendo discutido e problematizado, sendo alvo de críticas quanto ao seu produto final - a atuação profissional do médico. Isto porque, via de regra, a atuação profissional do médico não atende às demandas que envolvem uma série de desafios e dilemas associados à relação médico-paciente, o atendimento humanizado, a comunicação, entre outros aspectos que tornam necessário uma formação para além do conhecimento técnico-científicobiológico. Estas críticas foram fundamentais para promover alterações currículares e fortalecer novos modos de compreender o processo de ensino-aprendizagem na área médica, sendo possível observar estudos e práticas que refletem um novo modo de compreender a educação médica e que buscam atender os desafios profissionais requeridos ao futuro profissional médico (NOGUEIRA, 2009; ARAGÃO; ALMEIDA, 2017).

Neste sentido, atualmente tem se discutido muito sobre quais metodologias pedagógicas seriam consideradas mais "adequadas" para a formação médica, lançando como desafio a necessidade de defrontar o aluno com situações práticas da atuação profissional médica, possibilitando ao discente vivenciar dilemas éticos, desenvolver a comunicação, a empatia, o conhecimento prático, entre outros aspectos com relação ao atendimento em saúde, articulado ao conhecimento técnico-científico (FEUERWERKER, 2006; SIQUEIRA, 2006).

Além disso, a publicação das Diretrizes Curriculares Nacionais do Curso de Graduação em Medicina (DCN), em 2014, deu um passo importante em direção à reflexão sobre o ensino médico, ao impactar, provocar e desafiar as instituções de ensino superior a promover a formação de um perfil profissional diferenciado. Segundo a DCN, espera-se que as instituições de ensino superior formem o profissional médico com perfil generalista, humanista, crítico e reflexivo. Acredita-se que, com este perfil, o profissional seja capaz de atuar com base em princípios éticos no atendimento à saúde e doença, em seus diferentes níveis de atenção, promovendo a prevenção, recuperação e reabilitação no processo de saúde, com responsabilidade e compromisso social, tendo em vista a saúde integral do ser humano (BRASIL. MINISTÉRIO DA EDUCAÇÃO. CONSELHO NACIONAL DE EDUCAÇÃO, 2014) . 
No entanto, haja vista a tamanha complexidade deste perfil profissional, ancorado no desenvolvimento de um conjunto de competências, habilidades e atitudes, as instituições de ensino superior vêm cada vez mais repensando e discutindo suas práticas e propostas de ensino. Desde suas discussões iniciais na década de 1950, o campo da Educação Médica vem crescendo por meio da criação de programas de pós-graduação, periódicos específicos da área, eventos nacionais e internacionais, e sociedades (HAMAMOTO FILHO et al., 2013).

Desta forma, tendo em vista o expressivo e crescente número de publicações na área de Educação Médica, torna-se necessário o seguinte questionamento: Quais são os temas pesquisados no Brasil no âmbito da graduação médica? Neste sentido, este estudo teve como objetivo realizar uma revisão integrativa da literatura a fim de mapear os principais temas estudados na área da Educação Médica em nível de graduação, possibilitando traçar as principais tendências e propostas pedagógicas para a formação do profissional médico no Brasil.

\section{METODOLOGIA}

Trata-se de uma revisão integrativa da literatura, conduzida de maneira sistemática, buscando sintetizar as evidências encontradas em um resumo significativo da literatura disponível. Esta pesquisa foi realizada de acordo com as diretrizes publicadas pelo University of York's Centre for Reviews and Dissemination (DISSEMINATION; YORK, 2009).

\section{Estratégia de busca}

Para a localização das publicações realizou-se uma busca eletrônica na base de dados Google Acadêmico, utilizando os descritores "educação médica" e "educação de graduação em medicina”, mediante consulta prévia ao vocabulário DeCS (Descritores em Ciência da Saúde). 


\section{Critérios de elegibilidade}

Para a seleção dos estudos, adotaram-se os seguintes critérios: (i) estudos com metodologia quantitativa, qualitativa ou mista; (ii) publicados em português; (iii) que avaliaram o tema da educação médica no Brasil, no ambito da graduação, com data de publicação a partir de 01/01/2013.

Foram excluídos da seleção os seguintes estudos: (i) não disponíveis na íntegra; (ii) livros; (iii) dissertações; (iii) teses; (iv) carta ao editor; (v) resumos de anais; (vi) publicações duplicadas; e (vii) editorial.

\section{Desfecho da busca}

A pesquisa inicial identificou 264 artigos potenciais para inclusão. A análise das publicações transcorreu em duas etapas. A primeira foi realizada pelos autores, de forma independente, utilizando-se de um processo em "funil", excluindo as publicações que não correspondiam aos crítérios de inclusão, com base nos títulos e resumos de todos os artigos identificados na busca eletrônica. Em seguida, foi realizado comparação entre os estudos selecionados pelos autores. Em caso de discórdia, optou-se pela não exclusão da publicação. Após esta etapa, as publicações que compuseram a amostra final foram examinadas integralmente.

\section{Abstração e síntese dos dados}

Como foram encontrados estudos quantitativos e qualitativos na presente revisão, a abordagem narrativa foi o método mais adequado para identificar temas e analisar os resultados das publicações examinadas. Para tanto, utilizou-se a abordagem de análise temática, descrita por Braun e Clarke (2006), para a construção de um texto narrativo pelos dois autores envolvidos na análise e síntese dos dados. Sendo assim, após a análise e com base nos temas identificados, os artigos foram classificados nas seguintes categorias: a arte mobilizando o ensino; o processo de avaliação; a formação e as percepções de docentes; o uso de tecnologias digitais no ensino médico; as percepções de alunos sobre o ensino médico; metodologias ativas de ensino-aprendizagem; relatos de experiência de instituições de ensino médico sobre a reformulação curricular; relatos de experiência sobre a reformulação de disciplinas; e o repensar sobre a educação médica. 


\section{RESULTADOS}

Foram localizadas 264 publicações incluindo artigos não disponíveis na íntegra, livros, dissertações, teses, carta ao editor, resumos de anais, publicações duplicadas e editorial. Destas, 171 foram excluídas por não atender aos critérios de inclusão, resultado em 93 publicações como amostra final. (Tabela 1 - ANEXO 1)

$\mathrm{Na}$ Tabela 2 é apresentado a distribuição das publicações identificadas na busca eletrônica realizada de 2013 a 2018, de acordo com as categorias criadas e ano de publicação. Por meio desta, é possível observar uma queda expressiva no número de publicações no período entre 2015 e 2016, seguido por retomada crescente e constante do número de publicações.

Tabela 2 - Distribuição das publicações identificadas na busca eletrônica realizada de 2013 a 2018, de acordo com as categorias e ano de publicação.

\begin{tabular}{|c|c|c|c|c|c|c|c|}
\hline Categoria, $n$ & 2013 & 2014 & 2015 & 2016 & 2017 & 2018 & $\begin{array}{c}\text { Total de } \\
\text { publicações/categoria }\end{array}$ \\
\hline A arte mobilizando o ensino & 1 & 1 & 0 & 1 & 0 & 1 & 4 \\
\hline $\begin{array}{l}\text { As percepções de alunos } \\
\text { sobre o ensino médico }\end{array}$ & 1 & 1 & 0 & 2 & 2 & 6 & 12 \\
\hline $\begin{array}{l}\text { A formação e as percepções } \\
\text { de docentes }\end{array}$ & 0 & 0 & 0 & 0 & 2 & 2 & 4 \\
\hline $\begin{array}{l}\text { Metodologias ativas de } \\
\text { ensino-aprendizagem }\end{array}$ & 0 & 7 & 0 & 1 & 4 & 6 & 18 \\
\hline O processo de avaliação & 3 & 0 & 1 & 2 & 0 & 1 & 7 \\
\hline $\begin{array}{c}\text { O uso de tecnologias digitais } \\
\text { no ensino médico }\end{array}$ & 2 & 3 & 2 & 1 & 3 & 4 & 15 \\
\hline $\begin{array}{c}\text { Relatos de experiência de } \\
\text { instituições de ensino médico } \\
\text { sobre a reformulação } \\
\text { curricular }\end{array}$ & 1 & 3 & 0 & 1 & 1 & 3 & 9 \\
\hline $\begin{array}{l}\text { Relatos de experiência sobre a } \\
\text { reformulação de disciplinas }\end{array}$ & 0 & 1 & 0 & 0 & 4 & 6 & 11 \\
\hline $\begin{array}{c}\text { O repensar sobre a educação } \\
\text { médica }\end{array}$ & 1 & 4 & 1 & 1 & 3 & 3 & 13 \\
\hline Total de publicações/ano & 9 & 20 & 4 & 9 & 19 & 32 & 93 \\
\hline
\end{tabular}

Fonte: Própria. 
Quando se considerou o número percentual de publicações, as categorias que apresentaram menor índice (< 10\%) foram: relatos de experiência de instituições de ensino médico sobre a reformulação curricular (9.7\%); o processo de avaliação (7.5\%); a arte mobilizando o ensino (4.3\%); e a formação e as percepções de docentes (4.3\%). (Figura 1)

Figura 1: Distribuição percentual das publicações identificadas na busca eletrônica realizada de 2013 a 2018, de acordo com a categoria atribuída.

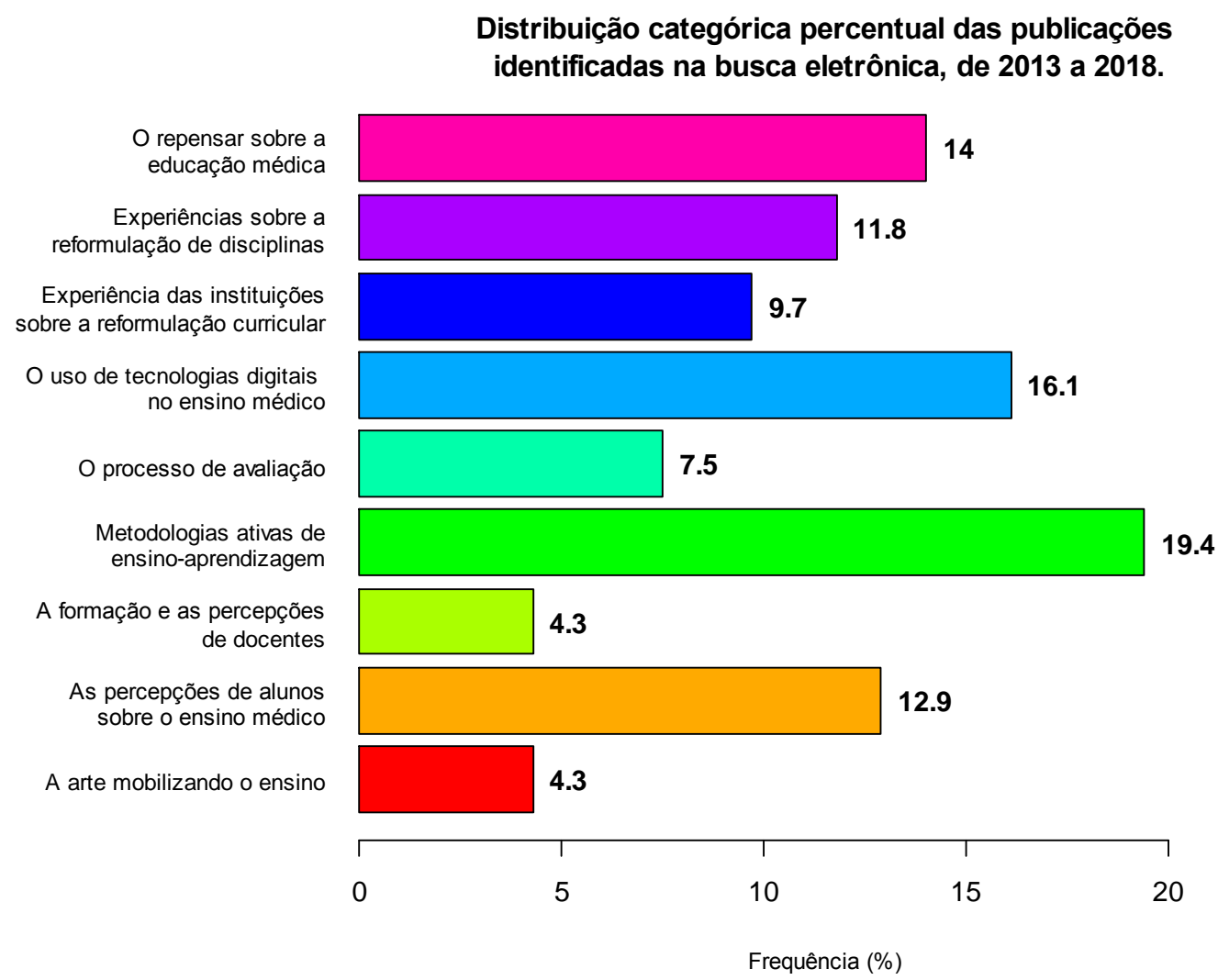

Fonte: Própria.

\section{DISCUSSÃO}

Ao mapear os principais temas estudados na área da Educação Médica ao nível da graduação, nota-se um crescimento constante nos últimos dois anos do número de publicações, concentradas principalmente em temas relacionados à criação de novas estratégias de ensino.

A categoria Metodologias Ativas de Ensino-Apredizagem foi construída com base nos estudos que abordaram a utilização de metodologias ativas na prática da educação médica. 
Tal forma de conduzir o processo de ensino e aprendizagem tem-se destacado como tendência de estratégia nas instituições de ensino superior, o que reflete o predomínio deste tema nesta revisão.

Neste aspecto, entende-se por metodologias ativas as estratégias de ensinoaprendizagem em que o aluno é o protagonista de seu desenvolvimento profissional, buscando romper o paradigma do professor como sendo único detentor do saber. Neste tipo de metodologia de ensino, estimula-se o confronto do aluno com situações teórico-práticas capazes de mobilizar suas funções cognitivas por meio de diferentes recursos, destacandose na presente revisão as seguintes estratégias: aprendizagem baseada em equipes(BOLLELA et al., 2014), simulação realística(AQUINO; SMANIA, 2018; BRANDÃO; COLLARES; MARIN, 2014; DOURADO; GIANNELLA, 2014; FLORES; BEZ; BRUNO, 2014; LUNA; SPIGHT, 2014; MOTTA; BARACAT, 2018; PAZIN-FILHO; CARLOTTI; SCARPELINI, 2017; SCHWELLER et al, 2018), aprendizagem baseada em problemas(CARABETTA JR, 2016; CARABETTA, 2017; CAVALCANTE et al., 2018a; MAIA, 2014), ensino participativo(FERREIRA, 2017; SILVA, 2018), uso de narrativas(CLARO; MENDES, 2018; GROSSMAN; CARDOSO, 2014), estudos dirigidos(FREITAS, 2017) e avaliação crítica da literatura médica(SILVA, 2018).

Em seguida, a categoria Uso de Tecnologias Digitais no Ensino foi criada a partir da identificação do uso de ferramentas digitais no ensino médico. Trata-se de uma tendência global em expansão no âmbito educacional, impactando também na formação médica. Neste aspecto, foram identificadas diversas formas de aplicação destas ferramentas digitais: o uso de telemedicina (ALMINO et al, 2014), a criação de ambientes virtuais de aprendizagem (AZENHA et al., 2017; DA SILVA et al, 2017; DINIZ JUNIOR et al, 2014; GOUDOURIS; GIANNELLA; STRUCHINER, 2013; SILVA et al., 2015; VASCONCELOS; VASCONCELOS, 2013; VILAS BÔAS MAGALHÃES; TEIXEIRA FERNANDES; MIN LI, 2014), aprendizagem hibrica (GOUDOURIS; STRUCHINER, 2015), uso de tecnologias móveis(CASTRO; MARÇAL, 2016; PEREIRA; KUBRUSLY; MARÇAL, 2017), utilização de redes sociais (Facebook (BEDÊ; ARAGÃO, 2018), Youtube (BARBOSA et al., 2018) e blogs (MENDONÇA; FONSECA, 2018)), e o uso de ferramenta de comunicação (WhatsApp) (BARBOSA et al., 2018; PAULINO et al., 2018).

Em Repensando a Educação Médica, contemplou-se as publicações de natureza teórica, que ressaltam a importância de se (re) pensar o processo de educação médica, com a justificativa de que o ensino tecnicista e biologizante não é suficiente para a formação do perfil profissional do médico em nossa atual conjuntura social. Neste aspecto, os seguintes 
temas emergiram como objeto de discussão: o desenvolvimento do pensamento crítico(ARAGÃO; ALMEIDA, 2017), a importância da integralidade na educação médica (MAKUCH; ZAGONEL, 2017; WENCESLAU; RÖHR; TESSER, 2014), o ensino de terapias alternativas (TEIXEIRA, 2017), a construção da autonomia (CHIARELLA et al., 2015; SMOLKA et al., 2013), reflexões sobre a educação médica (RANGEL, 2014; SILVA; BARACAT, 2016; SILVEIRA; STELET; PINHEIRO, 2014), o desenvolvimento da liderança (PROVENZANO et al., 2014), o papel da tutoria no processo de ensino-aprendizagem (MARTINS; FALBO NETO; SILVA, 2018), e a atuação em cuidados paliativos (BLASCO, 2018; CALDAS; MOREIRA; VILAR, 2018).

As categorias até então apresentadas vão de encontro com a necessidade de novas estratégias e concepções de ensino, de modo a favorecer o desenvolvimento do perfil profissional médico proposto pelas Diretrizes Curriculares. Contudo, com menor número de publicações encontram-se as categorias que refletem a análise do impacto destas inovações sobre o cotidiano de docentes e o resultado na aprendizagem discente.

Na categoria Percepção de Alunos, as publicações buscaram investigar a percepção dos alunos sobre algumas das propostas de ensino utilizadas pelas instituições e disciplinas. Neste sentido, foram encontrados investigações sobre a satisfação em relação ao curso e metodologia (MOURA et al., 2018; SMOLKA; GOMES; SIQUEIRA-BATISTA, 2014; USCOCOVICH, 2017), preferência por caso reais ou fictícios (PEREIRA et al., 2016), a prática do feedback(PRICINOTE; PEREIRA, 2016), a percepção sobre a relação médico-paciente (BIBERGSALUM et al., 2018; NASCIMENTO et al., 2018), a necessidade de auto-cuidado na prática profissional (CIRINO; DE MORAES; HIGA, 2017), a percepção dos alunos sobre as competências pedagógicas dos docentes (BELFOR et al., 2018), e a avaliação do ensino das disciplinas de semiologia médica (COSTA et al., 2018), embriologia(RIBEIRO, 2018) e anatomia(REIS et al., 2013).

Em Relato de Experiência das Instituições de Ensino Na Reformulação Curricular, a experiência na realização de alterações e criação de novas propostas curriculares foi relatada por instituições públicas e privadas, quais foram: Universidade Estadual do Sudoeste da Bahia (BRANDÃO; ROCHA; SILVA, 2013), Univerisdade Federal do Sul da Bahia (ALMEIDA FILHO et al., 2014, 2018), uma universidade filantrópica no sul do Brasil(FRANCO; CUBAS; FRANCO, 2014), Universidade de Brasilia (SOBRAL, 2014), Universidade de São Paulo (SOBRAL, 2014), Universidade Federal de Minas Gerais (SAVASSI; DIAS; GONTIJO, 2018; 
SOBRAL, 2014), uma universidade pública na cidade de Sobral/CE (TEÓFILO; SANTOS; BADUY, 2016), Universidade Estadual de Santa Cruz (COUTO et al., 2018) e Universidade Federal do Rio Grande do Norte (OLIVEIRA et al., 2017).

De forma similar, a categoria Relato de Experiência na Reformulação de Disciplinas, concentrou publicações que apresentaram a experiência na inovação do ensino de diferentes áreas, quais foram: saúde coletiva (DA SILVA; RAMALHO; DE SOUZA, 2018; VILLELA et al., 2017), clínica médica (KAHWAGE NETO et al., 2017; LOPES et al., 2018; PEIXOTO; SANTOS; FARIA, 2018), medicina da família (MAGALHÃES; BELMONTE; LUNA, 2014), cuidados paliativos (MALTA; RODRIGUES; PRIOLLI, 2018), ciências morfofuncionais(MATOS, 2017), homeopatia(OLIVEIRA et al,, 2018), e disciplinas integradoras (CAVALCANTE et al., 2018b; DE SOUZA et al., 2018).

Não obstante, uma vez que as propostas de ensino sofreram transformações, tornase necessário (re) pensar o método de avaliação discente. Este aspecto foi abordado na categoria Processo de Avaliação (CAMARGO; GEMPERLI; AULER JUNIOR, 2018; GONTIJO et al., 2013; MEDEIROS et al., 2013; MEGALE et al., 2015; PASTURA; SANTORO-LOPES, 2013; TRONCON, 2016; ZEPPONE et al., 2016) Neste aspecto, destacou-se a descrição do processo de elaboração de uma matriz de avaliação em ensino médico, em que diferentes domínios são avaliados como: profissionalismo, relacionamentos interpessoais e comunicação, atenção integral à saúde da pessoa, organização de sistemas de saúde e atenção em saúde pública, e gestão de conhecimento (GONTIJO et al., 2013). Outros aspectos relevantes foram a avaliação do desenvolvimento de competências afetivas e empáticas em discentes (MEDEIROS et al,, 2013) e a percepção de docentes sobre o método de avaliação formativa (MEGALE et al., 2015).

Em A Arte Mobilizando o Ensino, agrupou-se as publicações que utilizaram diferentes formas de arte como estratégia de ensino, quais foram: cinema (SÁ; TORRES, 2013), vídeos (PASTOR JUNIOR et al., 2016; ROSEVICS et al., 2014) e literatura (SANTOS; LINS; MENEZES, 2018). Os estudos citados demonstram o potencial dessas formas de expressões artísticas em afetar o aluno, mobilizando suas emoções e pensamentos na interface com o atendimento em saúde.

Além da atenção à práticas e estratégias de ensino que visam afetar o aluno no processo de aprendizado, torna-se fundamental também compreender os afetos que 
permeiam a atuação e prática docente, visto que em um contexto de reformulações uma série de dilemas são impostos à sua ação. Via de regra, docentes precisam ensinar e desenvolver competências nos alunos de modo distinto daquele com que foi ensinado e construído em seu processo de formação. Portanto, torna-se fundamental compreender as percepções de docentes e discutir sua formação no âmbito de novas práticas de ensino.

Neste aspecto, a categoria Formação e Percepção de docentes apresenta os estudos que buscaram investigar a percepção dos professores sobre: a proposta humanística (VILAS BOAS et al., 2017), sua avaliação realizada por alunos(BARRETO; XAVIER; SONZOGNO, 2018), o processo de avaliação formativa (PORTELLA et al., 2017), e o relato de sua experiência em uma comunidade no sertão nordestino (VIEIRA; PINTO; MELO, 2018). Porém, com relação a esta categoria ressalta-se a presença de poucos estudos acerca da percepção e processo de formação docente, objetivando uma série de desafios para a área da Educação Médica.

Neste sentido, a partir da análise das diversas categorias apresentadas, é possível constatar que a área da Educação Médica possui uma ampla variedade de temas investigados, mas que há expressiva concentração de estudos relacionados à temas como o uso de tecnologias e metodologias ativas. Portanto, com este trabalho buscou-se demonstrar os temas que possuem mais estudos e também aqueles que precisam ser investigados, mapeando as tendências e projetando perspectivas para estudos futuros acercas de temáticas que não são privilegiadas na área atualmente.

\section{CONSIDERAÇõES FINAIS}

Este estudo permitiu o mapeamento dos principais temas de estudo na área de Educação Médica. Neste aspecto, o uso de tecnologias digitais mobilizando o ensino médico e as metodologias ativas de ensino-aprendizagem demonstrou ser objeto de destaque como estratégias de ensino.

Há uma tendência de criação de novas estratégias de ensino, porém faltam estudos que avaliem os impactos da implementação destas estratégias sob a perspectiva de docentes e discentes. Em consonância, as categorias que envolveram a percepção de docentes e discentes foram aquelas que apresentaram menor número de estudos. Portanto, a inclusão 
da percepção de docentes e discentes sobre as inovações no âmbito da educação médica devem se constituir como novas perspectivas de estudo à serem desenvolvidos na área.

Adicionalmente, ao se propor rompimentos de paradigmas no ensino médico, ressignificando o papel de docentes e discentes neste processo, alterando toda a estrutura curricular e a forma como o processo de ensino-aprendizagem é conduzido, torna-se imprescindível discutir o processo de avaliação. Assim, este tema também emerge como nova perspectiva de estudo a ser desenvolvida na área.

Neste sentido, conclui-se que a despeito do crescente número de publicações, concentradas na criação de novas estratégias de ensino que buscam renovar as estruturas curriculares e o processo de ensino-aprendizagem, a área de Educação Médica carece de estudos que buscam acessar o impacto dessas inovações sob a perspectiva de docentes, discentes e no processo de avaliação.

No que se refere às limitações do presente estudo, destaca-se a não abrangência de estudos internacionais, os quais ampliariam a discussão apresentada no cenário mundial, constituindo-se como desafios para os próximos estudos. Ainda, apesar dos descritores utilizados para a localização das publicações abrangerem, genericamente, o tema educação de graduação em medicina, as publicações que não utilizaram estes indexadores foram excluídas da análise, reforçando assim a importância do uso correto de descritores nas publicações da área de educação médica.

\section{REFERÊNCIAS}

ARAGÃO, J. C. S.; ALMEIDA, L. D. S. Raciocínio Clínico e Pensamento Crítico: Desenvolvimento na educação médica. Revista de Estudios e Investigación en Psicología y Educación, n. 12, p. 19, 17 dez. 2017.

BRASIL. MINISTÉRIO DA EDUCAÇÃO. CONSELHO NACIONAL DE EDUCAÇÃO. Resolução CNE/CES no 3, de 20/06/2014. Diretrizes Curriculares Nacionais do Curso de Graduação em Medicina. Diário Oficial da União, 2014.

BRAUN, V.; CLARKE, V. Using thematic analysis in psychology. Qualitative Research in Psychology, v. 3, n. 2, p. 77-101, jan. 2006.

DIAS, D. et al. O que se "ensina" no Ensino Superior: avaliando conhecimentos, competências, valores e atitudes. Revista Meta: Avaliação, v. 10, n. 29, p. 318, 15 ago. 2018.

DISSEMINATION, C. FOR R. AND; YORK, U. OF. Systematic Reviews: CRD's Guidance for Undertaking Reviews in Health Care. [s.l.] Centre for Reviews and Dissemination, 2009. 
FEUERWERKER, L. C. M. O movimento mundial de educação médica: as conferências de Edinburgh. Cadernos da Abem, v. 2, n. 2, p. 30-38, june 2006.

HAMAMOTO FILHO, P. T.; SANTOS FILHO, C. A.; ABBADE, J. D.; PERACOLI, J. C.. Produção científica sobre educação médica no Brasil: estudo a partir das publicações da Revista Brasileira de Educação Médica. Revista Brasileira de Educação Médica, v. 37, n. 4, p. 477-482, dez. 2013.

NOGUEIRA, M. I. As Mudanças na educação médica brasileira em perspectiva: reflexões sobre a emergência de um novo estilo de pensamento. Revista Brasileira de Educação Médica, v. 33, n. 2, p. 262-270, june 2009.

SIQUEIRA, B. P. CINAEM - um pouco de sua história. Cadernos da Abem, v. 2, n. 2, p. 54-56, june 2006. 


\section{ANEXO 1}

Tabela 1 - Resultado da busca bibliográfica.

\begin{tabular}{|c|c|c|c|c|c|}
\hline Autores / Categoria & Título & Periódico & Ano & Método & Local \\
\hline \multicolumn{6}{|l|}{ A arte mobilizando o ensino } \\
\hline SÁ, E. C.; TORRES, R. A.T. & Cinema como recurso de educação em promoção da saúde & Revista de Medicina & 2013 & Qualitativo & São Paulo, SP \\
\hline $\begin{array}{l}\text { ROSEVICS, L.; AGUIAR, D. A.; BORGES, C. R.; HASEGAWA FILHO, R.; } \\
\text { YAMASHITA, T. S.; MANCHAK, A. C.; AZEVEDO, V. F. }\end{array}$ & ProCura - a arte da Vida: um Projeto pela Humanização na Saúde & Revista Brasileira de Educação Médica & 2014 & Qualitativo & Curitiba, PR \\
\hline $\begin{array}{l}\text { PASTOR JUNIOR, A. DE A.; REZENDE FILHO, L. A. C. DE; PEREIRA, M. } \\
\text { V.; BASTOS, W. G. }\end{array}$ & Apropriações de filmes e vídeos na educação médica & Interfaces da Educação & 2016 & Qualitativo & Rio de Janeiro, RJ \\
\hline SANTOS, M. R. C.; LINS, L.; MENEZES, M. S. & "As intermitências da morte" no ensino da ética e bioética & Revista Bioética & 2018 & Qualitativo & Salvador, BA \\
\hline \multicolumn{6}{|l|}{ A formação e as percepções de docentes } \\
\hline $\begin{array}{l}\text { PORTELLA, M. B.; SABATHE, L. DA S.; IWASAKA-NEDER, J. B.; } \\
\text { IWASAKA-NEDER, P. L. }\end{array}$ & Avaliação formativa sob a ótica do tutor & Interdisciplinary Journal of Health Education & 2017 & $\begin{array}{c}\text { Quantitativ } \\
\quad\end{array}$ & Belém, PA \\
\hline VILAS BOAS, L. M.; DALTRO, M. R.; GARCIA, C. P.; MENEZES, M. S. & Educação médica: desafio da humanização na formação & Saúde em Redes & 2017 & Qualitativo & Salvador, BA \\
\hline BARRETO, N. A.P.; XAVIER, A. R. E. DE O.; SONZOGNO, M. C. & $\begin{array}{l}\text { Percepção de Tutores quanto a Sua Avaliação pelos Discentes de um } \\
\text { Curso Médico }\end{array}$ & Revista Brasileira de Educação Médica & 2018 & Qualitativo & Montes Claros, MG \\
\hline VIEIRA, R. M. DE M.; PINTO, T. R.; MELO, L. P. DE. & $\begin{array}{l}\text { Narrativas e Memórias de Docentes Médicos sobre o Ensino Baseado } \\
\text { na Comunidade no Sertão Nordestino }\end{array}$ & Revista Brasileira de Educação Médica & 2018 & Qualitativo & Caicó, RN \\
\hline \multicolumn{6}{|l|}{ O processo de avaliação } \\
\hline $\begin{array}{l}\text { GONTIJO, E. D.; ALVIM, C.; MEGALIS, J;; MELO, J. R. C.; LIMA, M. E. C. } \\
\text { DE C. }\end{array}$ & $\begin{array}{l}\text { Matriz de Competências Essenciais para a Formação e Avaliação de } \\
\text { Desempenho de Estudantes de Medicina }\end{array}$ & Revista Brasileira de Educação Médica & 2013 & Misto & Belo Horizonte, MG \\
\hline $\begin{array}{l}\text { MEDEIROS, N. S.; SANTOS, T. R. DOS; TRINDADE, E. M. V.; ALMEIDA, } \\
\text { K. J. Q. DE. }\end{array}$ & $\begin{array}{l}\text { Avaliação do Desenvolvimento de Competências Afetivas e } \\
\text { Empáticas do Futuro Médico }\end{array}$ & Revista Brasileira de Educação Médica & 2013 & Qualitativo & Brasilia, DF \\
\hline
\end{tabular}




\section{Pott e Pott Jr.}

PASTURA, P. S. V. C.; SANTORO-LOPES, G.

O Aprendizado Melhorado por Provas

MEGALE, L.; RICAS, J.; GONTIJO, E. D.; MOTA, J. A. C.

Percepcões e Sentimentos de Professores de Medicina frente Avaliação dos Estudantes - um Processo

Solitário

\section{Revista Brasileira de Educação Médica}

Revista Brasileira de Educação Médica

Estruturação de Sistemas para Avaliação Programática do Estudante Revista Brasileira de Educação Médica de Medicina

Relato de Experiência da Construção de uma Proposta para Competências

CAMARGO, C. P.; GEMPERLI, R.; AULER JUNIOR, J. O.C.
Revista Brasileira de Educacão Médica

Revista de Medicina
Medical education assessment Avaliação da Prática Profissional de um Curso Orientado por

2013 Qualitativo

Rio de Janeiro, RJ

2015 Qualitativo

Belo Horizonte, MG

2016

Qualitativo

Ribeirão Preto, SP

2016 Qualitativo

São Carlos, SP

Qualitativo

São Paulo, SP

\section{Relatos de experiência de instituições de ensino médico sobre a reformulação curricular}

\begin{tabular}{|c|c|c|}
\hline BRANDÃO, E. R. M.; ROCHA, S. V.; SILVA, S. S. DA. & $\begin{array}{l}\text { Práticas de Integração Ensino-Serviço-Comunidade: Reorientando a } \\
\text { Formação Médica }\end{array}$ & Revista Brasileira de Educação Médica \\
\hline $\begin{array}{l}\text { ALMEIDA FILHO, N. DE; SANT ANA, L. A. A.; SANTOS, V. P. DOS; } \\
\text { COUTINHO, V.; LOUREIRO, S. }\end{array}$ & $\begin{array}{l}\text { Formação Médica na UFSB: I. Bacharelado Interdisciplinar em Saúde } \\
\text { no Primeiro Ciclo }\end{array}$ & Revista Brasileira de Educação Médica \\
\hline FRANCO, C. A. G. DOS S.; CUBAS, M. R.; FRANCO, R. S. & $\begin{array}{l}\text { Currículo de Medicina e as Competências Propostas pelas Diretrizes } \\
\text { Curriculares }\end{array}$ & Revista Brasileira de Educação Médica \\
\hline SOBRAL, D. T. & $\begin{array}{l}\text { Três Casos de Inovação Curricular no Panorama recente (1964-1988) } \\
\text { da Educação médica Brasileira: Subsídios de um retrospecto Baseado } \\
\text { na revisão De Documentos }\end{array}$ & Revista Brasileira de Educação Médica \\
\hline
\end{tabular}

OLIVEIRA, A. L. DE O. E; MELO, L. P. DE; PINTO, T. R.; AZEVEDO, G. D. DE; SANTOS, M. DOS; CAMARA, R. B. G. DA; COSTA, P. M. DA; MATA, A. N. DES.

Vivência integrada na comunidade: inserção longitudinal no Sistema de Saúde como estratégia de formação médica

Interface - Comunicação, Saúde, Educação

Jequié, BA

14 Qualitativo

Itabuna, BA

2014 Qualitativo

Curitiba, PR

2014 Qualitativo

Brasilia, DF

TEÓFILO, T. J. S.: SANTOS, N. L. P. DOS; BADUY, R. S.

Apostas de mudança na educação médica: trajetórias de uma escola Interface - Comunicação, Saúde, Educação de medicina

Revista Docência do Ensino Superior

Dermação Médica, Atenção Primerária E Intererdiscia Sobre Articulaçôes Necessárias

Revista Brasileira de Educação Médica

COUTO, V. B. M.; SANTOS, C. M. B.; SAMPAIO, B. P.; DE ALMEIDA, I. Vivenciando a Rede: Caminhos para a Formação do Médico no S.; MEDEIROS, S. C.; SANTOS, N. G. S.; MENEZES, T. A. M.; SANTOS, 
ALMEIDA FILHO, N. DE; LOPES, A. A. COUTINHO D. M. CARDOSO, A. J.; SANTANA, L. A.; SANTOS, V. P. DOS; GUIMARAES, L. H. Formação Médica na UFSB: III. Aprendizagem Orientada po Problemas e Competências

Revista Brasileira de Educaç̃o Médica

2018 Qualitativo

Itabuna, BA

\section{Relatos de experiência sobre a reformulação de disciplinas}

MAGALHÃES, T. N.; BELMONTE, T. DE S. A.; LUNA, C. DE A. A Medicina de Família na Educação Médica: Um Núcleod Atenção Terciária para a Aprendizagem em Atenção

Primária

MATOS, M.P.

Metodologias ativas auxiliando no aprendizado das ciências mofologa a tiva auxiliando no aprénizado das clências

CAVALCANTE, T.; MELO, B.; BATISTA, R.; JORDAO, D.; BESERRA, K. ANDRADE, L; LIMA JUNIOR, R. BOMFIM, A.

\section{Uma Experiência De Integração Ensin}

Alunos Do Curso De Graduação Em

Medicina Na Atenção Básica No Município De Maceió-Al, Brasil

KAHWAGE NETO, S. G.; BRAGA, T. K. K.; PORTELLA, M. B. ANDRIOLO, R. B.

The Teaching of Clinical skills and the Applicability of a Simplified Guide to Physical Examination in Undergraduate Medical Training

W. A.D.DE ALMEIDA,W.S., DEOLIVEIRA, F. M.

Educação em saúde: agentes com

Ofcinas de Planejamento em Saúde na Disciplina de Saúde Coletiva: Relato de Experiência

DA SILVA, R. E. B. G.; RAMALHO, A. DE P. Q.; DE SOUZA, C. C. O.

The Process of Clinical Reasoning among Medical Students

LOPES,

Paradigma na Formação Médica: Atitudes e Conhecimentos de

Acadêmicos sobre Morte e Cuidados Paliativos

MALTA, R.; RODRIGUES, B.; PRIOLLI, D. G.

OLIVEIRA, I. F. DE; PLUSO, B. H. B.; FREITAS, F. A. C.; NASCIMENTO, Homeopatia na Graduação Médica: Trajetória da Universidade OLIVETRA, Fomeopatia na Gradure

Processos de Desenvolvimento do Raciocínio Clínico em Estudantes de Medicina

PEIXOTO, J. M.SANTOS, S. M. E. FARIA, R. M. D. DE.
VILLELA, E. F. DE M.; BASTOS, L. K.; DUTRA, G. G.; DO NASCIMENTO, Edu
Revista de Ciências Médicas e Biológicas

Revista Ciência Plural

Revista Brasileira de Educaç̃̃o Médica

Revista Eletrônica de Comunicação, Informação e Inovação em

Grad+ Revista de Graduação USP

Revista Brasileira de Educação Médica

Revista Brasileira de Educação Médica

Revista Brasileira de Educação Médica

Revista Brasileira de Educação Médica
2013 Qualitativo

Rio de Janeiro, RJ 
DE SOUZA, M. C. A. CORTES, P.P. DE R R RABELLO, E. DA COSTA, E. M. A.; CORTES JUNIOR, J. C. DE S.; MENDONÇA, M. A.

As percepções de alunos sobre o ensino médico

REIS, C.; MARTINS M. de M.; MENDES R. A. F.; GONÇALVES L. B.;

SAMPAIO FILHO H. C.; MORAIS M. R.; OLIVEIRA S. E. B.

Avaliação da Percepção de Discentes do Curso Médico acerca do Estudo Anatômico

GUIMARÃES A. L. S.

SMOLKA, M. L. R. M. GOMES, A. P.; SIQUEIRA-BATISTA, R.

Autonomia no Contexto Pedagógico: Percepção de Estudantes de Medicina acerca da Aprendizagem Baseada em Problemas

PEREIRA, A. T.; GALLIAN, D. M. C.; REGINATO, V.; BENEDETTO, M. A. Percepção de Alunos de Medicina com a Dramatização: uma

C. DE.

Experiência Pedagógica

PRICINOTE, S. C. M. N.; PEREIRA, E. R. S.

Percepção de Discentes de Medicina sobre o Feedback no Ambiente de Aprendizagem

BELFOR, J. A. SENA, I. S. SILVA, D. K. B. DA. LOPES, B. T. S. KOGA JUNIOR, M. SANTOS, B. R. F. DOS.

Competências pedagógicas docentes sob a percepcão de alunos de medicina de universidade da Amazônia brasileira

CIRINO, A. A. DE O. G.; DE MORAES, M. A. A.; HIGA, E. DE F. R.

USCOCOVICH, V.S. M.

CUIDADO INTEGRAL NA FORMAÇ̃̃o MÉDICA: percursos e desafios

A avaliação da aprendizagem sob a ótica de acadêmicos de medicin

BIBERG-SALUM, T. G.F FOLETTO, L. D · SANTOS, M. F. C. ANDRADE,

L.P. DE; BENTO, L. M. A.

Desenvolvimento e Avaliação de Competências Atitudinais no

Contexto de Diferentes Metodologias para os Cursos de Medicina

COSTA, G. P. O.; HERCULANO, T. B.; GAMA, A. L. H.; CABRAL, R. P.;

CAMPOS, R. B.; DE OLIVEIRA, D. N. S.

MOURA, D. T. D. DE. FOLTRAN, R. S. FRAIZ I C. NOVAK, E. M.

Articulação Entre os Ciclos Básico e Profissionalizante: Percepção dos Alunos da UFPR.

NASCIMENTO, G. M.; ALMEIDA JUNIOR, S. L. DE; SILVA, A. M. T. C.; $\quad$ Avaliação da Relação Médico-Paciente em Alunos Internos de um CARVALHO, I. G. M. DE; ALMEIDA, R. J. DE.

Curso de Medicin

Testando novas metodologias de aprendizagem para o ensino de

embriologia humana: relato de experiência e percepção do
discentes
Revista Brasileira de Educação Médica

Revista Brasileira de Educação Médic

Revista Brasileira de Educação Médica

Revista Brasileira de Educação Médica

Ciência \& Saúde Coletiva

Revista da Universidade Vale do Rio Verde

spaço para a Saúde - Revista de Saúde Pública do Parana

Revista de Ensino, Educação e Ciências Humanas

Revista Brasileira de Educação Médica

Revista Brasileira de Educação Médic

Revista Brasileira de Educação Médica

Revista Brasileira de Educação Médica
2013 Quantitativ

2014 Qualitativo

Teresópolis, RJ

2016 Qualitativo

São Paulo, SP

2016 Qualitativo

Goiânia, GO

2017 Qualitativo

Macapá, AP

2017 Qualitativo

Marília, SP

2017 Qualitativo

Curitiba, PR

2018 Quantitativ Campo Grande, MS

2018 Qualitativo

João Pessoa, PB

$2018 \quad$ Misto

Curitiba, PR

2018 Quantitativ

2018 Qualitativo
Goiania, GO

Salvador, BA 
O repensar sobre a educação médica

\begin{tabular}{|c|c|c|c|c|c|}
\hline $\begin{array}{l}\text { SMOLKA, M. L. R. M.; GOMES, A. P.; COTTA, R. M. M.; SANTANA, L. } \\
\text { A.; BATISTA, R. S. }\end{array}$ & $\begin{array}{l}\text { A autonomia pedagógica do estudante: perspectivas na educação } \\
\text { médica }\end{array}$ & Ensino, Saúde e Ambiente & 2013 & Qualitativo & Viçosa, MG \\
\hline $\begin{array}{l}\text { PROVENZANO, B. C.; FERREIRA, D. V.; MACHADO, A. P. G.; ARANHA, } \\
\text { R. N. }\end{array}$ & Liderança na educação médica & Revista Hospital Universitário Pedro Ernesto & 2014 & Qualitativo & Rio de Janeiro, RJ \\
\hline RANGEL, M. & Dimensões da educação médica & Revista Hospital Universitário Pedro Ernesto & 2014 & Qualitativo & Rio de Janeiro, RJ \\
\hline SILVEIRA, R. P.; STELET, B. P.; PINHEIRO, R. & $\begin{array}{l}\text { Crise na educação médica? Um ensaio sobre o referencial } \\
\text { arendtiano }\end{array}$ & Interface - Comunicação, Saúde, Educação & 2014 & Qualitativo & Acre, $\mathrm{AC}$ \\
\hline WENCESLAU, L. D.; RÖHR, F.; TESSER, C. D. & $\begin{array}{l}\text { Contribuições da medicina antroposófica à integralidade na } \\
\text { educação médica: uma aproximação hermenêutica }\end{array}$ & Interface - Comunicação, Saúde, Educação & 2014 & Qualitativo & Viçosa, $M G$ \\
\hline $\begin{array}{l}\text { CHIARELLA, T.; BIVANCO-LIMA, D.; MOURA, J. DE C.; MARQUES, M. } \\
\text { C. DA C.; MARSIGLIA, R. M. G. }\end{array}$ & $\begin{array}{l}\text { A Pedagogia de Paulo Freire e o Processo Ensino-Aprendizagem na } \\
\text { Educação Médica }\end{array}$ & Revista Brasileira de Educação Médica & 2015 & Qualitativo & São Paulo, SP \\
\hline BLASCO, P. G. & $\begin{array}{l}\text { A ordem dos fatores altera o produto. Reflexões sobre educação } \\
\text { médica e cuidados paliativos }\end{array}$ & Educación Médica & 2016 & Qualitativo & São Paulo, SP \\
\hline SILVA, L. F. F. DA; BARACAT, E. C. & Medical education - historic perspective and future challenges & Revista de Medicina & 2016 & Qualitativo & São Paulo, SP \\
\hline ARAGÃO, J. C. S.; ALMEIDA, L. D. S. & $\begin{array}{l}\text { Raciocínio Clínico e Pensamento Crítico: Desenvolvimento na } \\
\text { educação médica }\end{array}$ & Revista de Estudios e Investigación en Psicología y Educación & 2017 & Qualitativo & Rio de Janeiro, RJ \\
\hline MAKUCH, D. M. V.; ZAGONEL, I. P. S. & $\begin{array}{l}\text { A integralidade do Cuidado no Ensino na Área da Saúde: uma } \\
\text { Revisão Sistemática }\end{array}$ & Revista Brasileira de Educação Médica & 2017 & Qualitativo & Curitiba, PR \\
\hline TEIXEIRA, M. Z. & $\begin{array}{l}\text { Panorama mundial da educação médica em terapêuticas não } \\
\text { convencionais }\end{array}$ & Revista de Homeopatia & 2017 & Qualitativo & São Paulo, SP \\
\hline CALDAS, G. H. DE O.; MOREIRA, S. DE N. T.; VILAR, M. J. & Palliative care: A proposal for undergraduate education in Medicine & Revista Brasileira de Geriatria e Gerontologia & 2018 & Qualitativo & Natal, RN \\
\hline MARTINS, A. C.; FALBO NETO, G.; SILVA, F. A. M. DA. & $\begin{array}{l}\text { Características do Tutor Efetivo em ABP - Uma Revisão de } \\
\text { Literatura }\end{array}$ & Revista Brasileira de Educação Médica & 2018 & Qualitativo & Boa Vista, RR \\
\hline
\end{tabular}

Revista SUSTINERE, Rio de Janeiro, v. 7, n.1, p. 132-152, jan-jun, 2019 
VASCONCELOS, D. F. P.; VASCONCELOS, A. C. C. G.
Tecnologias de informação e comunicação e ensino semipresencia na educação médica

Desenvolvimento de um ambiente virtual de ensino em histologia para estudantes da saúde ALMINO, M. A. F. B.; RODRIGUES, S. R.; BARROS, K. S. B.; FONTELES,
A. S.; ALENCAR, L. B. L.; LIMA, L. L. DE; JORGE, M. S. B.

Telemedicina: um instrumento de educação e promoção da saúde pediátrica

DINIZ JUNIOR, J.; FERNANDES BERTIM, M.; VALENTIM, R. DE M.; DINIZ $R$.

O Grupo Especial De Interesse Em Educação Médica Como Estratégia Jornal Brasileiro de TelesSaúde Facilitadora Da Aprendizagem Colaborativa À Distância Para O Profissionais Da Saúde

VILAS BÔAS MAGALHÃES, L.; TEIXEIRA FERNANDES, P.; MIN LI, L. C Como ensinar epilepsia na graduação médica? Uma proposta da Comissão de Ensino da LBE para a educação médica via web

SILVA, J. R. DA; MEDEIROS, F. B. DE; MOURA, F. M. S. DE; BESSA, W. DA S.; BEZERRA, E. L. M.

Uso das Tecnologias de Informação e Comunicação no Curso de Medicina da UFRN

GOUDOURIS, E.; STRUCHINER, M.

Aprendizagem Híbrida na Educação Médica: uma Revisão Sistemática

CASTRO, E.; MARÇAL, E.

Uma Análise sobre o Uso das Tecnologias Móveis para a Educação Médica

AZENHA, E.; CUNHA, G.; COSTA, V. C. R.; STREIT JUNIOR, R.; BENFICA PAZ L : JOSE, A. BEZERRA, C.

Ações de educação médica a distância por meio de protótipos de apresentações eletrônicas interativas

Desenvolvimento, Utilização e Avaliação de uma Aplicação Móvel para Educação Médica: um Estudo de Caso em Anestesiologia

DA SILVA, M. H. L. F.; RODRIGUES, A. J. DA S.; CORREIA NETO, J. D A S.; VILAR, G.; BARBOSA, R. F.; DE BRITO ROCHA, M. F.

Aplicação das Plataformas de Stewart na produção de Objetos de Aprendizagem em 3D para a educação médica

BEDÊ, P. V.; ARAGÃO, L. P.

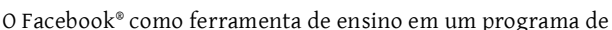
monitoria

MENDONÇA, N.; FONSECA, N.

O blog como ferramenta auxiliar no ensino-aprendizagem da semiologia médica

WhatsApp ${ }^{\oplus}$ como Recurso para a Educação em Saúde: Contextualizando Teoria e Prática em um Novo Cenário de Ensino-
Revista Brasileira de Educação Médic

Revista Brasileira de Educação Médica

Revista Brasileira de Educação Médica

Journal of Epilepsy and Clinical Neurophysiology

Revista Brasileira de Educação Médica

Revista Brasileira de Educação Médica

Revista Sistemas e Mídias Digitais

Revista de Medicina e Saúde de Brasília

Revista Novas Tecnologias na Educação

Revista ESPACIOS

Revista de Saúde Digital e Tecnologias Educacionais

International Journal of Health Education

Revista Brasileira de Educação Médica
2013 Qualitativo

2013 Qualitativo

2014 Qualitativo

2014 Qualitativo

2015 Qualitativo

2015 Qualitativo

2016 Qualitativo

2017 Qualitativo

2017 Qualitativo

2017 Quantitativ

2018 Qualitativo

2018 Qualitativo

2018 Qualitativo
Rio de Janeiro, RJ

Parnaíba, PI

Fortaleza, CE

Natal, RN

Campinas, SP

Rio de Janeiro, RJ

Rio de Janeiro, RJ

Fortaleza, CE

Brasilia, DF

Fortaleza, CE

Recife, PE

Forotaleza, CE

Salvador, BA

Uberlândia, MG 
Aprendizagem

BARBOSA, S. E. da S.; DO CARMO, L. A.; MEDEIROS, R. L. S. F. M de; CABRAL, S. A. A. de O. LOPES, R. E. M.; ALVES, M. N. T.

Impacto na Rotina Acadêmica pela Utilização de Novas por Estudantes de Medicina do Semiárido Paraibano
2018 Qualitativo

Cajazeiras, $\mathrm{PB}$

Metodologias ativas de ensino-aprendizagem

BOLLELA, V. R.; SENGER, M. H.; TOURINHO, F. S. V.; AMARAL, E.

Aprendizagem baseada em equipes: Da teoria a pratica

A simulação realística co
estudantes de medicina

DOURADO, A. S. S.; GIANNELLA, T. R.

FLORES, C. D.; BEZ, M. R.; BRUNO, R. M.

GROSSMAN, E.; CARDOSO, M. H. C. A.

LUNA, R. A.; SPIGHT, D.

MAIA, J.A.

CARABETTA JR, $\mathrm{V}$.

CARABETTA JR, V.

FERREIRA, D. S.

FREITAS, E. D. DE

PAZIN-FILHO, A. CARLOTTI, A.P. D. C.P. SCARPELINI, S.

AQUINO, R. B.; SMANIA, M. C. análise das percep̧̧ões de alunos e professores de um Hospital do$$
\text { Rio de Janeiro }
$$

O Uso de Simuladores no Ensino da Medicin

A narrativa como ferramenta na educação médica

Simulação em educação médica: uma mudança necessária

Metodologias problematizadoras em currículos de graduaç̃o médic

Metodologia ativa na educação médica

Metodologia da problematização: possibilidade para aprendizagem significativa e interdisciplinar na educacao medic

Ensino participativo na educação médica

Manifesto pelos cuidados paliativos na graduação em medicina: estudo dirigido da Carta de Praga

Implementation and development of the Simulation Laboratory (SimLab) of Ribeirao Preto Medical School of University of Sao Paulo (SimLab) of RPMS-USP)

Uso da simulação no ensino da anestesiologia em escola de medicina Scientia Medica
Ensino baseado em simulação na formação continuada de médicos: Revista Brasileira de Educação Médica

Medicina (Brazil)

Scientia Medica

Revista Brasileira de Informática na Educação

Revista Hospital Universitário Pedro Ernesto

Revista Hospital Universitário Pedro Ernesto

Revista Brasileira de Educação Médica

Revista de Medicina

FEM: Revista de la Fundación Educación Médica

Arte Médica Ampliada

Revista Bioética

Medicina (Ribeirao Preto)

2014 Qualitativo

2014 Qualitativo

2014 Qualitativo

Ribeirão Preto, SP

São Paulo, SP

Rio de Janeiro, RJ

Porto Alegre, RS

Rio de Janeiro, RJ

Rio de Janeiro, R.

São Caetano do Sul, SP

São Paulo, SP

São Paulo, SP

2017 Qualitativo

2017 Qualitativo

Não informado

2017 Qualitativo

Salvador, BA

2017 Qualitativo

Ribeirão Preto, SP

2018 Qualitativo 


\section{Pott e Pott Jr.}

\begin{tabular}{|c|c|c|c|c|c|}
\hline $\begin{array}{l}\text { CAVALCANTE, A. N.; LIRA, G. V.; CAVALCANTE NETO, P. G.; LIRA, R. } \\
\text { G. M. }\end{array}$ & $\begin{array}{l}\text { Análise da Produção Bibliográfica sobre Problem-Based Learning } \\
\text { (PBL) em Quatro Periódicos Selecionados }\end{array}$ & Revista Brasileira de Educação Médica & 2018 & Qualitativo & Sobral, CE \\
\hline CLARO, L. B. L.; MENDES, A. A. A. & $\begin{array}{l}\text { Uma experiência do uso de narrativas na formação de estudantes de } \\
\text { Medicina }\end{array}$ & Interface - Comunicação, Saúde, Educação & 2018 & Qualitativo & Niterói, RJ \\
\hline MOTTA, E. V. DA; BARACAT, E. C. & $\begin{array}{l}\text { Treinamento de habilidades cirúrgicas para estudantes de medicina } \\
\text { - papel da simulação }\end{array}$ & Revista de Medicina & 2018 & Qualitativo & São Paulo, SI \\
\hline $\begin{array}{l}\text { SCHWELLER, M.; ROVEIRO, D. L.; PASSERI, S. R.; WAND ERLEY, J. S.; } \\
\text { CARVALHO-FILHO, M. A. }\end{array}$ & $\begin{array}{l}\text { Simulated medical consultations with standardized patients: In- } \\
\text { depth debriefing based on dealing with emotions }\end{array}$ & Revista Brasileira de Educação Médica & 2018 & Qualitativo & Campinas, $\mathrm{SI}$ \\
\hline SILVA, F.E. & $\begin{array}{l}\text { A Avaliação Crítica da Literatura Médica como Instrumento de } \\
\text { Complementação Educacional no Internato de Medicina }\end{array}$ & Revista Brasileira de Educação Médica & 2018 & Qualitativo & Macaé, RJ \\
\hline
\end{tabular}

Fonte: Própria.

(c) $\mathrm{Br}$

Este trabalho está licenciado com uma Licença Creative Commons - Atribuição 4.0 Internacional. 